Stains of authenticity

Angew. Chem. Int. Ed. http://doi.org/f2sp7w (2014)

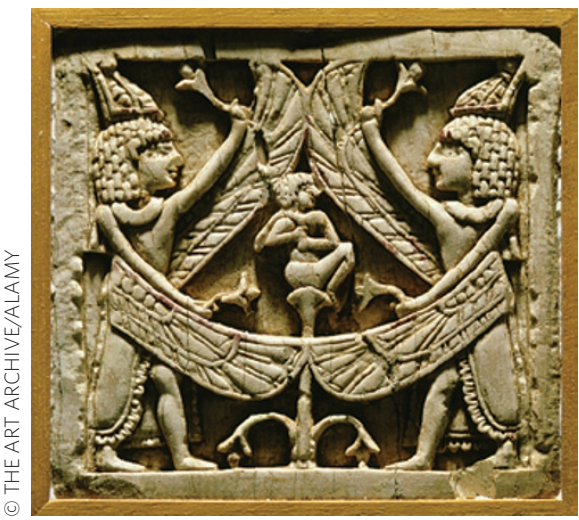

The phoenician ivory carvings found at Arslan Tash in northern Syria date back to the end of the ninth century $\mathrm{BC}$, and are renowned for their artistic quality. For archaeological conservationists, they also represented something of a conundrum. The carvings were originally covered with a thin layer of gold foil, and over the centuries this has reacted with the ivory to give rise to distinctive purple stains. Detailed chemical analyses carried out at the Louvre museum in Paris revealed that these stains consist of gold nanoparticles (AuNPs) with a relatively uniform size distribution. Ivory is, in essence, a collagen-based mineralized tissue with a high specific surface area arising from the presence of hydroxyapatite nanocrystals, and it is known that AuNPs can be made by photoreducing collagen- $\mathrm{HAuCl}_{4}$ complexes in solution. But their formation in archaeological finds had not been examined. Now, Ina Reiche and colleagues demonstrate that the collagen can indeed act as a stabilizer for AuNP formation in a simple reaction that occurs at ambient burial conditions. With the origin of the stains established, the authors propose they can be used as a marker of authenticity for other gold-plated ivory artefacts found in the future.

\section{Getting more from photons Nature Commun. 5, 4148 (2014)}

As a first step to generate free charge-carriers in a solar cell, photons impinging on the device must promote electrons from the valence band to the conduction band of the absorbing material. This means that the energy of the photons must be equal or higher than a threshold value corresponding to the material energy bandgap. The energy in excess of this threshold is usually lost in rapid thermal relaxation of the excited charges; yet it may be used to promote additional electrons via the process of carrier multiplication. Victor Klimov and colleagues now show that PbSe nanocrystals surrounded by a thick CdSe shell are ideal candidates to recycle the excess photon energy into additional free charges. Indeed, a photon having energy slightly higher than twice the bandgap of the PbSe core has non-zero probability to excite two charge carriers. The researchers suggest that this enhanced carrier multiplication is due to reduced electronic coupling between the core and the shell states, which slows down the thermal relaxation of shell-localized charges.

$L M$

\section{Screened DNA packs faster Phys. Rev. Lett. 112, 248101 (2014)}

Despite being negatively charged, DNA can be compacted to almost crystalline densities inside virus capsids. However, how this is achieved has been debated for years. Most theoretical models predict that attractive DNA-DNA interactions - such as those resulting from the screening effect of nearby positively charged molecules - would facilitate DNA packaging by countering the effects of DNA self-repulsion and loss of

\title{
Aggregate prevention
}

The formation of cerebral amyloid fibrils as a result of the aggregation of amyloid $\beta$ peptides is indicative of Alzheimer's disease. Now, Linqi Shi and colleagues demonstrate the inhibition of amyloid $\beta$ fibrillation using mixed-shell polymeric micelles (MSPM). The micelles, formed from the self-assembly of two amphiphilic copolymers, have a poly(caprolactone) core and a mixed shell of polyethylene oxide and poly( $\mathrm{N}$-isopropylacrylamide) (PNIPAM). At physiological temperature, the PNIPAM chains collapse and form hydrophobic domains. These domains interact with the hydrophobic amyloid $\beta$ peptides and their oligomeric aggregates, and hence, inhibit the formation of fibrils. The ratio of polymers within the shell can be altered to tune the surface properties and influence amyloid $\beta$ fibrillation. Shi and colleagues also report that the proteolytic degradation of MSPM-amyloid $\beta$ peptide complexes is greater than that of mature amyloid fibrils. This combination of prevention of fibril formation by the binding of amyloid $\beta$ peptides and oligomeric species with the ability to degrade amyloid $\beta$ aggregates, results in the reduction of amyloid- $\beta$-mediated neurotoxicity in invitro studies. AS

entropy. Now, optical-tweezer experiments by Nicholas Keller and colleagues demonstrate that the packaging of DNA is accelerated in the presence of low concentrations of the positively charged polyamine spermidine. However, when the concentration of spermidine is sufficiently high to induce attractive interactions in the DNA, the molecular motors tend to slow and stall, drastically lowering the average packaging rate. The authors conclude that the screening of repulsive DNA-DNA interactions speeds up DNA compaction, but that attractive interactions induce the molecular motors to experience highly variable loads that cause the DNA to be packaged in a wider range of conformations, some of which can eventually impede the functioning of the motor.

\section{Complementary observables Phys. Rev. Lett. 112, 253602 (2014)}
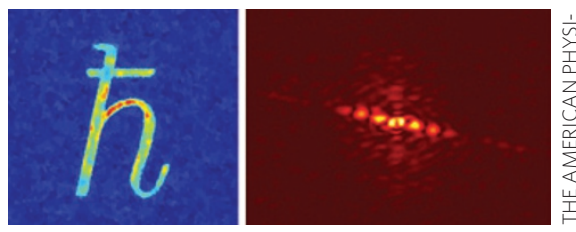

The Heisenberg uncertainty principle states that the simultaneous knowledge of complementary observables, such as position and momentum, of a quantum system will always reach a fundamental information limit; this means that the more precisely you measure one observable, the less knowledge you'll end up having about its complementary. Now, Gregory Howland and colleagues show how this fundamental limit of quantum mechanics can be bypassed in the measurement of the photons' position and momentum. Their method records information about the photons' momentum distribution directly from CCD (charge-coupled device) images. Before CCD measurements, the momentum information is slightly disturbed as a result of encountering a series of filtering masks. The role of this preCCD-filtering is to allow the filtered photon state to carry a small amount of information about the photons' position distribution. This information is then efficiently extracted by compressed sensing. Using this methodology the authors are able to recover both position and momentum information of four different objects. This result does not invalidate the Heisenberg uncertainty principle per se, but it does show how, by measuring a mixed signal of complementary observables, it is possible to use information efficiently. 